\title{
Rate of progression of cognitive decline in Alzheimer's disease: effect of butyrylcholinesterase $\mathrm{K}$ gene variation
}

\section{Holmes, C Ballard, D Lehmann, A David Smith, H Beaumont, I N Day, M Nadeem Khan, S Lovestone, M McCulley, C M Morris, D G Munoz, K O'Brien, C Russ, T Del Ser, D Warden}

See end of article for authors' affiliations

\section{Correspondence to:}

Dr C Holmes, University of

Southampton, Clinical

Neurosciences Research

Division, Memory

Assessment and Research

Centre, Moorgreen

Hospital, Botley Road,

Southampton; c.holmes@

soton.ac.uk

Received 13February 2004

Revised version received

25 August 2004

Accepted 25 August 2004
J Neurol Neurosurg Psychiatry 2005;76:640-643. doi: 10.1136/jnnp.2004.039321

Objective: To determine whether individuals with Alzheimer's disease (AD) and the $K$ variant allele of butyrylcholinesterase have a slower rate of cognitive decline than those without the $\mathrm{K}$ variant allele of butyrylcholinesterase.

Method: The cognitive status of 339 community based subjects with AD was assessed with the Mini Mental State Examination at baseline and yearly over a three year follow up period. The rates of cognitive decline of subjects with and without the $\mathrm{K}$ variant allele were compared.

Result: Presence of the K allele was associated with a slower average rate of cognitive decline in subjects with severe $A D$.

Conclusions: This finding is consistent with the suggestion that the $\mathrm{K}$ variant of butyrylcholinesterase has an important role in disease progression in $A D$, and this may have implications for treatment.

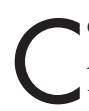
ognitive decline is, by definition, invariably present in Alzheimer's disease (AD). However, the rate of cognitive decline is highly variable among individuals, with some having a very benign course. ${ }^{12}$ An understanding of the aetiological factors underlying this variability is clearly very important because of the enormous potential benefits for the development of more effective therapeutic approaches. There are a number of reasons for the heterogeneity of cognitive decline, including non-subject variables such as inter and intra scoring inconsistencies and the non-linearity of rating scales, and subject variables including the presence of concurrent illness, the beneficial and detrimental effects of drug treatment, and genetic variation. ${ }^{3}$ Genetic variation is clearly important in the progression of other neurological diseases and might therefore be expected to account for a substantial proportion of the variance in the differential rates of cognitive decline in AD. However, this area has been relatively neglected. The most studied allele is apolipoprotein $\mathrm{E}(\mathrm{ApoE}) \in 4$, but this has not been associated with the rate of decline in most studies. ${ }^{4}$

Butyrylcholinesterase is expressed in most human tissues. In rodents, like acetylcholinesterase, it is involved in the breakdown and termination of acetylcholine. ${ }^{5}$ The activity of butyrylcholinesterase is elevated in $\mathrm{AD}$, probably as a result of the increasing numbers of plaques and tangles and has been hypothesised to contribute to cognitive decline in the later stages of the illness. ${ }^{67}$ The $\mathrm{K}$ variant of butyrylcholinesterase has a functional point mutation that changes Ala539 to threonine and reduces its molecular concentration in serum by $30 \%{ }^{8}$

In a preliminary study, the presence of the $\mathrm{K}$ variant was associated with a significantly reduced rate of progression of cognitive decline in individuals with moderate to severe dementia with Lewy bodies." A meta-analysis has also shown the $\mathrm{K}$ variant to be a risk factor for the development of $\mathrm{AD}$ in elderly men. ${ }^{10}$ We hypothesised that the $\mathrm{K}$ variant might be associated with different rates of disease progression in individuals with late onset $\mathrm{AD}$ and that these effects may be more prominent as the disease progresses. To examine fully the effects of butyrylcholinesterase $\mathrm{K}$ in $\mathrm{AD}$ subjects of both sexes from a wide age range with varying cognitive impairment at baseline we elected to combine two complementary UK community based cohorts: an Oxford cohort, a largely incident population with mild disease severity at baseline and a London cohort, a largely prevalent population with more severe disease at baseline.

\section{METHODS}

Subjects were recruited from two sites, the Institute of Psychiatry Camberwell Dementia Case Register (CDCR), London, and the Oxford Project to Investigate Memory and Ageing (OPTIMA), Oxford.

\section{London $(C D C R)$ clinical sample $(n=151)$}

Venous blood samples were obtained from individuals from the $\mathrm{CDCR}^{11}$ an inclusive, largely prevalent, community dwelling representative sample. All subjects fulfilled the National Institute of Neurological and Communicative Disorders and Stroke and the Alzheimer's Disease and Related Disorders Association (NINCDS-ADRDA) criteria $^{12}$ for probable or possible $\mathrm{AD}$ (probable $\mathrm{n}=105$, possible $\mathrm{n}=46$ ). Validation of the clinical diagnosis of $\mathrm{AD}$ against post mortem in this sample has been previously reported with high positive predictive values (0.89) against CERAD diagnostic criteria. ${ }^{13}$ The median age of the sample group was 82 years (range 30 years) and $120(80 \%)$ were women. Clinical diagnosis was made independently by two psychiatrists based on a checklist for symptoms of the disease with strict adherence to NINCDS-ADRDA criteria. Cognitive assessment was recorded by trained clinical research nurses using the Mini Mental State Examination (MMSE). ${ }^{14}$

Abbreviations: $A D$, Alzheimer's disease; ApoE, apolipoprotein $E_{\text {; }}$ CDCR, Camberwell Dementia Case Register; MMSE, Mini Mental State Examination; OPTIMA, Oxford Project to Investigate Memory and Ageing 
Table 1 Demographic and clinical details of the subjects

\begin{tabular}{lllll}
\hline Variable & $\begin{array}{l}\text { London cohort } \\
(\mathbf{n}=151)\end{array}$ & $\begin{array}{l}\text { Oxford cohort } \\
(\mathbf{n}=188)\end{array}$ & $\begin{array}{l}\text { Combined } \\
(\mathbf{n}=339)\end{array}$ & $\begin{array}{l}\text { Between cohorts } \\
\text { statistical tests }\end{array}$ \\
\hline No of men (\%) & $31(21)$ & $96(51)$ & $127(37)$ & $\chi^{2} p<0.0001$ \\
Age in years, median (range) & $82(30)$ & $75(38)$ & $79(45)$ & MWU $p<0.0001$ \\
Age at onset in years, median (range) & $79(29)$ & $72(42)$ & $76(45)$ & MWU $p<0.0001$ \\
Baseline MMSE score, median (range) & $11(28)$ & $22(23)$ & $18(30)$ & MWU $p<0.0001$ \\
Annual MMSE decline, median (range) & $-2.0(22)$ & $-2.3(21)$ & $-2.3(22)$ & MWU p=0.09 \\
\hline MMSE, Mini Mental State Examination; MWU, Mann-Whitney U test. & & \\
\hline
\end{tabular}

Assessment followed a standardised protocol to maximise interrater reliability. All subjects were followed up in their homes by the same rater at yearly intervals, for a period of up to three years with repeat MMSE on each occasion. None of the subjects was prescribed cholinesterase inhibitors during the course of the study.

All subjects were Caucasian and genotyped blind to clinical data. Ethical approval was obtained from the Bethlem Royal and the Maudsley hospitals and the Institute of Psychiatry ethical committee.

\section{Oxford (OPTIMA) clinical sample ( $n=188$ )}

Venous blood samples were obtained from individuals from the Oxford region recruited into the OPTIMA cohort, ${ }^{15}$ an inclusive, largely incident, community dwelling sample. All subjects fulfilled the NINCDS-ADRDA criteria ${ }^{12}$ for probable or possible $\mathrm{AD}$ (probable $\mathrm{n}=117$, possible $\mathrm{n}=71$ ). The median age of the sample group was 75 years (range 38 ) and $92(49 \%)$ were women. Clinical diagnosis was made independently by a psychiatrist and neurologist based on a checklist for symptoms of the disease with strict adherence to NINCDS-ADRDA criteria. A total of 60 subjects were confirmed as having "definite" or "probable" AD at autopsy by CERAD criteria. ${ }^{16}$ In this cohort, the positive predictive value has been found previously ${ }^{17}$ to be $100 \%$ for NINCDS$\mathrm{ADRDA}$ "probable $\mathrm{AD}^{\prime}$ and $68 \%$ for "possible $\mathrm{AD}^{\prime}$ against CERAD criteria. Cognitive assessment was recorded by trained clinical research nurses using the MMSE. Assessment followed a standardised protocol to maximise interrater reliability. All subjects were followed up at yearly intervals, for a period of up to three years or more with repeat MMSE on each occasion. None of the subjects was prescribed cholinesterase inhibitors during the course of this study.

All subjects were Caucasian and genotyped blind to clinical data. Ethical approval was obtained from the Oxford ethical committee.

\section{Genotyping}

Venous blood samples were genotyped for the $\mathrm{K}$ variant of butyrylcholinesterase and the $\epsilon 4$ allele of ApoE following the same protocols for both study centres as described elsewhere. ${ }^{18} 19$

\section{Statistical analysis}

Assessment of normality of the variables age, age of onset, baseline MMSE, and MMSE decline was determined by

Table 2 Genotype frequencies of the K variant of butyrylcholinesterase. Data are $\mathrm{n}(\%)$

\begin{tabular}{lllll}
\hline Genotype & $\begin{array}{l}\text { London } \\
(\mathbf{n}=151)\end{array}$ & $\begin{array}{l}\text { Oxford } \\
(\mathbf{n}=188)\end{array}$ & $\begin{array}{l}\text { Combined } \\
(\mathbf{n}=339)\end{array}$ & $\begin{array}{l}\text { Between cohorts } \\
\text { statistical tests }\end{array}$ \\
\hline${ }^{*}{ }^{*}$ & $94(0.62)$ & $124(0.66)$ & $218(0.64)$ & $\chi^{2} 0.5 p=0.8$ \\
$K /{ }^{*}$ & $50(0.33)$ & $56(0.30)$ & $106(0.31)$ & \\
$\mathrm{K} / \mathrm{K}$ & $7(0.05)$ & $8(0.04)$ & $15(0.05)$ & \\
\hline
\end{tabular}

quantile-quantile (Q-Q) plots of the residuals. Non-parametric comparisons of outcome variables by cohort site were performed using the Mann-Whitney $U$ test. Genotype frequencies of the cohorts were compared using $\chi^{2}$ analysis. Spearman's rank correlation test was used for correlation between non-parametric variables.

The rate of cognitive decline was based on the average slope of MMSE points change per year. Differences in the average annual MMSE decline in the whole group by the presence or absence of the $\mathrm{K}$ variant of butyrylcholinesterase were assessed by the Mann-Whitney $U$ test. The subjects were then grouped into four categories depending on their baseline MMSE scores $(>24 ; \leqslant 24$ and $>16 ; \leqslant 16$ and $>8$; $\leqslant 8$ points). Differences in the average annual MMSE decline in the four categories by the presence or absence of the $\mathrm{K}$ variant of butyrylcholinesterase was initially assessed by independent $t$ tests. Linear regression analysis with the average annual MMSE decline as the dependent variable was then used to assess for confounding and effect modification by the independent variables MMSE at baseline, age, age of onset, sex, and the presence or absence of ApoE $\epsilon 4$.

\section{RESULTS}

The demographic details of the subjects by cohort site are shown in table 1. The age, age at onset, baseline MMSE scores, and average overall MMSE decline of the separate and combined cohorts were assessed as being different from a normal distribution (Q-Q plot of residuals). The annual MMSE decline, however, when assessed by baseline MMSE groups did not appear different from a normal distribution either with or without the $\mathrm{K}$ variant allele (Q-Q plot of residuals)

The subjects enrolled from the Oxford (OPTIMA) cohort were significantly younger and had a younger age of onset (see table 1). They also had significantly less cognitive impairment at baseline and were more likely to be men than the London (CDCR) cohort. Although there was a trend towards a faster rate of cognitive decline in the Oxford group this was not significant (see table 1).

The subjects' genotype frequencies for the $\mathrm{K}$ variant of butyrylcholinesterase by cohort are shown in table 2 . There were no significant differences between the cohorts for the genotype frequencies for butyrylcholinesterase $\mathrm{K}$. The genotype frequencies for both cohorts were in Hardy-Weinberg equilibrium (Oxford $\chi^{2}=0.22, \mathrm{df}=1 \mathrm{p}=0.9$, London $\left.\chi^{2}=0.18, \mathrm{df}=1 \mathrm{p}=0.9\right)$.

Combining the two cohorts resulted in a group of 339 subjects. All had baseline MMSE scores with follow up MMSE at one year. In addition, 213 had MMSE follow up at two years and a further 143 had follow up at three years. Drop outs to follow up were largely due to death of the subjects; the characteristics of these subjects are detailed elsewhere. ${ }^{11} 15$

There was no significant association between median MMSE decline and the presence of the butyrylcholinesterase $\mathrm{K}$ allele (Mann-Whitney two tailed $\mathrm{U}$ test $\mathrm{p}=0.93$ ) in the combined cohort. In addition, there was no significant 


\begin{tabular}{|c|c|c|c|c|}
\hline & \multicolumn{4}{|l|}{ All subjects } \\
\hline & MMSE $>24$ & MMSE $\leqslant 24$ and $>16$ & MMSE $\leqslant 16$ and $>8$ & MMSE $\leqslant 8$ \\
\hline $\begin{array}{l}\text { K genotypes }(n) \\
\text { K allele absent }\left({ }^{*} / *\right)\end{array}$ & 66 & 123 & 93 & 57 \\
\hline $\begin{array}{l}\text { Mean MMSE decline (SE) } \\
\mathrm{n}(\%) \\
\mathrm{K} \text { allele present ( } / \mathrm{K} \\
\text { and } \mathrm{K} / \mathrm{K} \text { combined) }\end{array}$ & $\begin{array}{l}-2.4(0.5) \\
43(65)\end{array}$ & $\begin{array}{l}-3.1(0.4) \\
74(60)\end{array}$ & $\begin{array}{l}-2.6(0.4) \\
63(67)\end{array}$ & $\begin{array}{l}-1.8(0.3)^{*} \\
38(67)\end{array}$ \\
\hline $\begin{array}{l}\text { Mean MMSE decline (SE) } \\
\mathrm{n}(\%)\end{array}$ & $\begin{array}{l}-3.0(0.7) \\
23(35)\end{array}$ & $\begin{array}{l}-3.6(0.5) \\
49(40)\end{array}$ & $\begin{array}{l}-2.6(0.5) \\
30(33)\end{array}$ & $\begin{array}{l}-0.2(0.4) \\
19(33)\end{array}$ \\
\hline
\end{tabular}

association between median MMSE decline and age (Spearman's correlation $\left(r_{\mathrm{s}}\right)=0.05, \mathrm{p}=0.32$ ), age of onset $\left(r_{\mathrm{s}}=0, \mathrm{p}=1.0\right)$, sex (men -2.0 points $v$ women -2.3 points, Mann-Whitney $U$ test $p=0.75$ ), or presence of the ApoE $\epsilon 4$ allele ( $\epsilon 4$ positive -2.0 points $v \in 4$ negative -2.3 points, Mann-Whitney $U$ test $p=0.40$ ). There was a significant negative correlation between median MMSE decline and baseline MMSE examination $\left(r_{\mathrm{s}}=-0.14, \mathrm{p}=0.01\right)$. Table 3 shows the annual mean MMSE decline by baseline MMSE scores and by the presence or absence of the butyrylcholinesterase K allele for the combined cohort $(n=339)$.

There was no significant association between mean MMSE decline and the presence of butyrylcholinesterase $\mathrm{K}$ allele in the subject groups with baseline MMSE scores greater than 8 points. However, subjects carrying the K allele with a baseline MMSE less than or equal to 8 points had a significantly slower mean rate of cognitive decline (two tailed $t$ test $\mathrm{p}=0.002$; Bonferroni correction $\mathrm{p}=0.008$ ). There was a significant negative correlation between mean MMSE decline and baseline MMSE examination $\left(r_{\mathrm{s}}-0.50, \mathrm{p}<0.0001\right)$ in subjects with a baseline MMSE score of less than or equal to 8 points. No significant relations were found between age, age of onset, sex, or presence of the ApoE $\epsilon 4$ allele and mean MMSE decline in subjects with a baseline MMSE score of less than or equal to 8 points.

Linear regression analysis, both unweighted and weighted for number of years of follow up, with MMSE decline as the dependent variable and the presence or absence of the butyrylcholinesterase $\mathrm{K}$ allele and baseline MMSE score as independent variables, showed a reduced but still significant relation $(p=0.007$ unweighted and $p=0.016$ weighted $)$ between the presence of the $\mathrm{K}$ allele and a decreased rate of cognitive decline. There was no evidence for effect modification between MMSE scores at baseline and presence of the $\mathrm{K}$ allele. In addition, there was no evidence for confounding by the other variables of age, age of onset, sex, or presence of the ApoE $€ 4$ allele.

\section{DISCUSSION}

The younger age and milder cognitive impairment of the Oxford cohort at baseline compared with the London cohort is consistent with the selection of more incident cases in the Oxford cohort compared with the selection of more prevalent cases in the London cohort. Correcting for age and cognitive impairment resulted in a diminished, but still significantly, increased frequency of men in the Oxford cohort compared with the London cohort. The reasons for this are less clear but may reflect differences in the duration of illness between men and women. ${ }^{20}$

Butyrylcholinesterase $\mathrm{K}$ genotype frequencies were in Hardy-Weinberg equilibrium in both cohorts and were not significantly different between the two cohorts. In addition, the average median MMSE decline was not significantly different between the cohorts and followed similar estimates published elsewhere. ${ }^{31}$

The main finding of this study is that among subjects carrying the $\mathrm{K}$ allele of butyrylcholinesterase those with baseline MMSE scores of less than or equal to 8 points had a slower rate of cognitive decline. This relation does not appear to be confounded by the correlation between baseline MMSE scores and rate of cognitive decline. Likewise, other potential confounding factors such as age, age of onset, sex, and possession of the ApoE $\epsilon 4$ allele did not appear to account for this relation.

This study thus implies that the $\mathrm{K}$ variant of butyrylcholinesterase can influence disease progression. This is consistent with the proposed biological role of butyrylcholinesterase in plaque maturation and with the suggestion that its role becomes more important as the disease progresses due to its increased presence in plaques. ${ }^{22}$ This observation has important potential implications for treatment with respect to the possible benefits of agents such as cholinesterase inhibitors that inhibit butyrylcholinesterase as the disease severity progresses. It also offers the potential for development of other novel treatment approaches through mechanistic studies clarifying the role of butyrylcholinesterase.

\section{ACKNOWLEDGEMENTS}

The CDCR was supported by a number of staff over the five year period of data collection. The authors would specifically like to acknowledge J Leach, D McLoughlin, H Cadbury, R Pattison, F Garoghan, T Finnernan, C Armstrong, and M Ryde, and M Mulley for statistical advice. We are also grateful for the consistent cooperation of all medical and social agencies supplying information to the CDCR. We thank all patients and volunteers, members of OPTIMA and the Department of Neuropathology, Radcliffe Infirmary.

\section{Authors' affiliations \\ C Holmes, I N Day, M McCulley, University of Southampton, Southampton, UK \\ C Ballard, C M Morris, K O'Brien, Newcastle General Hospital, Newcastle-upon-Tyne, UK}

D Lehmann, A D Smith, H Beaumont, D Warden, OPTIMA, Department of Pharmacology, University of Oxford and Radcliffe Infirmary, Oxford, UK

S Lovestone, C Russ, M N Khan, The Institute of Psychiatry, London, UK D G Munoz, T Del Ser, Department of Neurology, Madrid, Spain and University of Western Ontario, Canada

This study was funded in part by Novartis Pharmaceuticals which has a special interest in the role of butyrylcholinesterase in the progression of Alzheimer's disease; Bristol Myers Squibb; the Norman Collisson Foundation; and by the Wellcome Trust.

Competing interests: $\mathrm{S}$ Lovestone has research collaborations and has received speaker's fees and educational grants from a variety of companies that manufacture cholinesterase inhibitors that may or may 
not have some butyrylcholinesterase inhibitor activity also. C Holmes has acted in a consultancy role and has obtained funds for research, and $C$ Ballard has acted in a consultancy role, received fees for speaking and has obtained funds for research, from Novartis, manufacturer of rivastigmine which markets a drug that has inhibitory properties on butyrylcholinesterase.

\section{REFERENCES}

1 Perrault A, Wolfson C, Egan $M$, et al. Prognostic factors for functional independence in older adults with mild dementia: results from the Canadian study of health and aging. Alzheimer Dis Assoc Disord 2002;16:42-7.

2 Holmes $\mathrm{C}$, Lovestone $\mathrm{S}$. Long term cognitive and functional decline in late onset Alzheimer's disease: therapeutic implications. Age Ageing 2003;32:200-4.

3 Corey BJ. The natural history of Alzheimer's disease. Dementia 2000;34:405-15.

4 Holmes C. Genotype and phenotype in Alzheimer's disease. Br J Psychiatry 2002;180:131-4

5 Giacobini E. Cholinesterase inhibitors: from the Calabar bean to Alzheimer therapy. In: Giacobini E, ed. Cholinesterases and Cholinesterase Inhibitors. London: Martin Dunitz, 2000.

6 Perry EK, Perry RH, Blessed G, et al. Changes in brain cholinesterases in senile dementia of Alzheimer type. Neuropathol Appl Neurobiol 1978;4:273-7.

7 Geula C, Mesulam MM. Cholinesterases and pathology of Alzheimer's disease. Alzheimer Dis Assoc Disord 1995:9:23-8.

8 Bartels CF, Jensen FS, Lockridge O, et al. DNA mutation associated with the butyrylcholinesterase $\mathrm{K}$-variant and its linkage to the atypical variant mutation and other polymorphic sites. Am J Hum Genet 1992;50:1086-103.

9 Perry E, McKeith I, Ballard C. The K variant of the butyrylcholinesterase enzyme is associated with significantly slower cognitive decline and predicts treatment response in dementia patients. Neurology 2003;10:1852-3.

10 Lehmann DJ, Williams J, McBroom J, et al. Using meta-analysis to explain the diversity of results in genetic studies of late-onset Alzheimer's disease and to identify high-risk subgroups. Neuroscience 2001;108:541-4.
11 Holmes C. The Camberwell dementia case register. Int I Geriatr Psychiatry 1996;11:369-75.

12 McKhann G, Drachman D, Folstein M, et al. Clinical diagnosis of Alzheimer's disease: report of the NINCDS-ADRDA Work Group under the auspices of Department of Health and Human Services Task Force on Alzheimer's disease. Neurology 1984;34:939-44

13 Holmes C, Cairns N, Lantos P, et al. The validity of current research diagnostic criteria for Alzheimer's disease, vascular dementia and dementia with Lewy bodies. Br J Psychiatry 1999:174:45-50.

14 Folstein MF, Folstein SE, McHugh PR. Mini Mental State: a practical method for grading the cognitive state of patients for the clinician. J Psychiatric Res 1975; 12:189-98

15 Jobst KA, Smith AD, Szatmari M, et al. Detection in life of confirmed Alzheimer's disease using a simple measurement of medial temporal lobe atrophy by computed tomography. Lancet 1992;340:1179-83.

16 Mirra SS, Heyman A, McKeel D, et al. The Consortium to Establish a Registry for Alzheimer's Disease (CERAD). 2. Standardization of the neuropathologic assessment of Alzheimer's disease. Neurology 1991;41:479-86.

17 Nagy Z, Esiri M, Hindley NJ, et al. Accuracy of clinical criteria for Alzheimer's disease in relation to different pathological diagnostic protocols. Dement Geriatr Cogn Disord 1998;9:219-26.

18 Jensen FS, Nielsen LR, Schwartz M. Detection of the plasma cholinesterase K variant by PCR using an amplification-created restriction site. Hum Hered 1996:46:26-31.

19 Wenham PR, Price WH, Blundell G. Apolipoprotein E genotyping by onestage PCR. Lancet 1991; 337:1158-9.

20 Burns $\mathbf{A}$, Lewis $\mathrm{G}$, Jacoby $\mathrm{R}$, et al. Factors affecting survival in Alzheimer's disease. Psychol Med 1991:21:363-70.

21 Clark-CM, Sheppard L, Fillenbaum G, et al. Variability in annual Mini-Mental State Examination score in patients with probable Alzheimer disease: a clinical perspective of data from the consortium to establish a registry for Alzheimer's disease. Arch Neurol 1999;56:857-62.

22 Mesulam MM, Geula C. Butyrylcholinesterase reactivity differentiates the amyloid plaques of aging from those of dementia. Ann Neurol 1994;36:722-7. 